\title{
The Pauli principle, QRPA and the two-neutrino double beta decay *
}

\author{
J. Schwieger, F. Šmkovic ${ }^{\dagger}$ and Amand Faessler \\ Institut für Theoretische Physik der Universität Tübingen, \\ Auf der Morgenstelle 14, 72076 Tübingen, Germany
}

(August 7, 2021)

\begin{abstract}
We examine the violation of the Pauli exclusion principle in the Quasiparticle Random Phase Approximation (QRPA) calculation of the two-neutrino double beta decay matrix elements, which has its origin in the quasi-boson approximation. For that purpose we propose a new renormalized QRPA with proton-neutron pairing method (full-RQRPA) for nuclear structure studies, which includes ground state correlation beyond the QRPA. This is achieved by using of renormalized quasi-boson approximation, in which the Pauli exclusion principle is taken into account more carefully. The full-RQRPA has been applied to two-neutrino double beta decay of ${ }^{76} \mathrm{Ge},{ }^{82} \mathrm{Se},{ }^{128} \mathrm{Te}$ and ${ }^{130} \mathrm{Te}$. The nuclear matrix elements have been found significantly less sensitive to the increasing strength of particle-particle interaction in the physically interesting region in comparison with QRPA results. The strong differences between the
\end{abstract}

\footnotetext{
*Supported by the "Deutsche Forschungsgemeinschaft", contract No. Fa 67/17-1 and by the "Graduierten Kolleg - Struktur und Wechselwirkung von Hadronen und Kernen", DFG, Mu 705/3.

${ }^{\dagger}$ On leave from:Bogoliubov Theoretical Laboratory, Joint Institute for Nuclear Research, 141980 Dubna, Moscow Region, Russia and Department of Nuclear Physics, Comenius University, Mlynská dolina F1, Bratislava, Slovakia
} 
results of both methods indicate that the Pauli exclusion principle plays an important role in the evaluation of the double beta decay. The inclusion of the Pauli principle removes the difficulties with the strong dependence on the particle-particle strength $g_{p p}$ in the QRPA on the two-neutrino double beta decay.

23.40.Hc

Typeset using REVTEX 


\section{INTRODUCTION}

Neutrinoless double beta decay $(0 \nu \beta \beta)$, which involves the emission of two electrons and no neutrinos, demands neutrino to be a Majorana particle and have a non-zero mass. This process violates the lepton number conservation and occurs in some theories beyond the standard model ( [1]- [5] for reviews). But, the $0 \nu \beta \beta$-decay has not yet been observed. The experimental lower limits on the half-lives provide the most stringent limits on the effective mass of the neutrinos and the parameters of the right-handed currents, depending on $0 \nu \beta \beta$ decay nuclear matrix elements. Two neutrino double beta decay $(2 \nu \beta \beta)$, which involves the emission of two antineutrinos and two electrons, is a second order weak process fully consistent with the standard model [1]- [5]. It is the rarest process observed so far in the nature. Direct measurements of $2 \nu \beta \beta$-decay half-lives provide a sensitive test of nuclear structure models. The reliable evaluation of the $2 \nu \beta \beta$-decay nuclear matrix elements is necessary to gain confidence in the calculated $0 \nu \beta \beta$-decay nuclear matrix elements.

The quasiparticle random phase approximation (QRPA) is the nuclear many-body method most widely used to deal with the nuclear structure aspects of the double beta decay process [6]- [16]. The QRPA has been found successful in explaining the quenching of the $0 \nu \beta \beta$-decay nuclear matrix elements and bring them into closer agreement with experimental values. But despite this success, the QRPA approach to double beta decay has some shortcomings. The main problem is that the results are extremely sensitive to the renormalization of the particle-particle component of the residual interaction, which is in large part responsible for suppressing calculated two neutrino decay rate. This feature has been confirmed in the calculations with both schematic contact $\delta$ interaction and realistic finite range two-body interaction. Several alterations of the QRPA have been proposed that might change that behavior including proton-neutron pairing [17, 18], higher order RPA corrections [19] and particle number projection [20,21]. However, none of these extension of the QRPA have changed the rapid variation of the calculated $2 \nu \beta \beta$-decay nuclear matrix elements with the increasing strength of the particle-particle force. 
The crucial point of the QRPA consists in the so-called quasi-boson approximation (QBA) leading to a violation of the Pauli exclusion principle. The validity of the QBA in the evaluation of the nuclear many body Green functions for double beta decay transitions is questioned because of the generation of too much ground state correlations with increasing strength of the particle-particle interaction leading to a collapse of the QRPA. The renormalized RPA [22]- [26] being proposed many years ago is a method going one step beyond the QBA. There are many variations of this method [27]- [31], which basically consists in replacing the uncorrelated ground state (Hartree-Fock ground state or BCS ground state) by the correlated ground state ( RPA or QRPA ground state) in the calculation of the expectation value of the commutator of two bifermion operators. The topic of this paper is to propose a renormalized QRPA with proton-neutron pairing and to study the influence of the ground state correlations beyond the QRPA on the value of $2 \nu \beta \beta$-decay matrix elements.

We note that recently Toivanen and Suhonen have proposed a similar method [32] to study the nuclear double beta decay which however does not take into account protonneutron pairing. Their method has been applied to calculate the two-neutrino double beta decay of ${ }^{100} \mathrm{Mo}$ by considering some of the ground state correlations beyond the QRPA.

\section{FORMALISM}

We describe the smearing of the Fermi surface by the Hartree- Fock- Bogoliubov (HFB) method, which includes proton-proton, neutron-neutron and proton-neutron pairing [17,18,33. Particle $\left(c_{\tau a m_{a}}^{+}\right.$and $\left.c_{\tau a m_{a}}, \tau=p, n\right)$ and quasiparticle $\left(a_{\mu a m_{a}}^{+}\right.$and $a_{\mu a m_{a}}$, $\mu=1,2)$ creation and annihilation operators for spherical shell-model states labeled by $\left(a m_{a}\right)$ are related to each other by the Bogoliubov transformation:

$$
\left(\begin{array}{c}
c_{p a m_{a}}^{+} \\
c_{n a m_{a}}^{+} \\
c_{p a \tilde{m}_{a}} \\
c_{n a \tilde{m}_{a}}
\end{array}\right)=\left(\begin{array}{cccc}
u_{a 1 p} & u_{a 2 p} & -v_{a 1 p} & -v_{a 2 p} \\
u_{a 1 n} & u_{a 2 n} & -v_{a 1 n} & -v_{a 2 n} \\
v_{a 1 p} & v_{a 2 p} & u_{a 1 p} & u_{a 2 p} \\
v_{a 1 n} & v_{a 2 n} & u_{a 1 n} & u_{a 2 n}
\end{array}\right)\left(\begin{array}{c}
a_{1 a m_{a}}^{+} \\
a_{2 a m_{a}}^{+} \\
a_{1 a \tilde{m}_{a}} \\
a_{2 a \tilde{m}_{a}}
\end{array}\right),
$$


where the tilde $\sim$ indicates the time reversed states $a_{\tau a \tilde{m}_{a}}=(-1)^{j_{a}-m_{a}} a_{\tau a-m_{a}}$. The label $a$ designates single particle (quasiparticle) quantum numbers $\left(n_{a}, l_{a}, j_{a}\right)$. The occupation amplitudes $u$ and $v$ and the single quasiparticle energies $E_{a \alpha}$ are obtained by solving the adequate HFB equation [17,18, 33]. The vacuum of the quasiparticle operators will be denoted by $\mid 0_{H F B}^{+}>$. We note that in the ground states of spherical even mass nuclei one can have only $\mathrm{J}=0$ and $\mathrm{T}=1$ proton-neutron pairs in the framework of the BCS and more general HFB theory. In principle one could have $\mathrm{T}=0, \mathrm{~J}=$ odd correlations but only for deformed nuclei [34]. The treatment here is restricted to spherical nuclei. Such deformation effects are taken effectively into account by renormalizing according to [17,18 the $\mathrm{T}=1, J=0^{+}$ proton-neutron pairing to be stronger than requested by the Brueckner reaction matrix elements of the Bonn potential. This strength is fitted to the experimental proton-neutron pairing gap extracted from the masses [17,18.

In the limit in which there is no proton-neutron pairing $u_{2 p}=v_{2 p}=u_{1 n}=v_{1 n}=0$. Then the HFB transformation in Eq. (1) reduces to two BCS transformations, first for protons $\left(u_{1 p}=u_{p}, v_{1 p}=v_{p}\right)$ and second for neutrons $\left(u_{2 n}=u_{n}, v_{2 n}=v_{n}\right)$.

We assume the nuclear motion to be harmonic and the phonon operator to be of the form [17, 18, 33

$$
\begin{gathered}
Q_{J M^{\pi}}^{m+}=\sum_{k l}\left\{X_{12}^{m}\left(k, l, J^{\pi}\right) A_{12}^{+}(k, l, J, M)+Y_{12}^{m}\left(k, l, J^{\pi}\right) \tilde{A}_{12}(k, l, J, M)\right\} \\
\quad+\sum_{\substack{k \leq l \\
\mu=1,2}}\left\{X_{\mu \mu}^{m}\left(k, l, J^{\pi}\right) A_{\mu \mu}^{+}(k, l, J, M)+Y_{\mu \mu}^{m}\left(k, l, J^{\pi}\right) \tilde{A}_{\mu \mu}(k, l, J, M)\right\},
\end{gathered}
$$

Here, $X^{m}$ and $Y^{m}$ are forward- and backward- going free variational amplitudes. The operator $A_{\mu \nu}^{+}(k, l, J, M)(\mu, \nu=1,2)$ creates a pair of quasiparticles coupled to angular momentum J with projection M, namely

$$
\begin{aligned}
A_{\mu \nu}^{+}(k, l, J, M) & =n(k \mu, l \nu) \sum_{m_{k}, m_{l}} C_{j_{k} m_{k} j_{l} m_{l}}^{J M} a_{\mu k m_{k}}^{+} a_{\nu l m_{l}}^{+}, \\
\tilde{A}_{\mu \nu}(k, l, J M) & =(-1)^{J-M} A_{\mu \nu}(k, l, J,-M)
\end{aligned}
$$

The factor $n(k \mu, l \nu)$ is a normalization in the case in which both quasiparticles are in the same shell: 


$$
n(k \mu, l \nu)=\left(1+(-1)^{J} \delta_{k l} \delta_{\mu \nu}\right) /\left(1+\delta_{k l} \delta_{\mu \nu}\right)^{3 / 2} .
$$

By using the machinery of the equation of motion we get eigenvalue equation

$$
\left(\begin{array}{ll}
\mathcal{A} & \mathcal{B} \\
\mathcal{B} & \mathcal{A}
\end{array}\right)_{J^{\pi}}\left(\begin{array}{c}
X^{m} \\
Y^{m}
\end{array}\right)_{J^{\pi}}=\Omega_{J^{\pi}}^{m}\left(\begin{array}{cc}
\mathcal{U} & 0 \\
0 & -\mathcal{U}
\end{array}\right)_{J^{\pi}}\left(\begin{array}{c}
X^{m} \\
Y^{m}
\end{array}\right)_{J^{\pi}},
$$

with the matrices

$$
\begin{aligned}
& \mathcal{A}_{J}\left(\mu k, \nu l ; \mu^{\prime} k^{\prime}, \nu^{\prime} l^{\prime}\right)=\left\langle 0_{R P A}^{+}\left|\left[A_{\mu \nu}(k, l, J, M),\left[\hat{H}, A_{\mu^{\prime} \nu^{\prime}}^{+}\left(k^{\prime}, l^{\prime}, J, M\right)\right]\right]\right| 0_{R P A}^{+}\right\rangle, \\
& \mathcal{B}_{J}\left(\mu k, \nu l ; \mu^{\prime} k^{\prime}, \nu^{\prime} l^{\prime}\right)=\left\langle 0_{R P A}^{+}\left|\left[A_{\mu \nu}(k, l, J, M),\left[\tilde{A}_{\mu^{\prime} \nu^{\prime}}\left(k^{\prime}, l^{\prime}, J, M\right), \hat{H}\right]\right]\right| 0_{R P A}^{+}\right\rangle,
\end{aligned}
$$

and the unitary-matrix $\mathcal{U}$

$$
\mathcal{U}=\left\langle 0_{R P A}^{+}\left|\left[A_{\mu \nu}(k, l, J, M), A_{\mu^{\prime} \nu^{\prime}}^{+}\left(k^{\prime}, l^{\prime}, J, M\right)\right]\right| 0_{R P A}^{+}\right\rangle .
$$

Here, $\hat{H}$ is the quasiparticle hamiltonian and $\Omega_{J^{\pi}}^{m}$ is the excitation energy $E_{m}^{J^{\pi}}-E_{0} . E_{m}^{J^{\pi}}$ and $E_{0}$ are respectively eigenenergies of the excited state $\mid J M_{m}^{\pi}>\left(\left|J M_{m}^{\pi}>=Q_{J M^{\pi}}^{m+}\right| 0_{R P A}^{+}>\right)$ and of the ground state $\mid 0_{R P A}^{+}>\left(Q_{J M^{\pi}}^{m} \mid 0_{R P A}^{+}>=0\right)$.

In order to solve the Eq. (5) we need additional approximation. In the framework of QBA

$$
\begin{aligned}
& {\left[A_{\mu \nu}(k, l, J, M), A_{\mu^{\prime} \nu^{\prime}}^{+}\left(k^{\prime}, l^{\prime}, J, M\right)\right]} \\
& \simeq\left\langle 0_{H F B}^{+}\left|\left[A_{\mu \nu}(k, l, J, M), A_{\mu^{\prime} \nu^{\prime}}^{+}\left(k^{\prime}, l^{\prime}, J, M\right)\right]\right| 0_{H F B}^{+}\right\rangle \\
& =n(k \mu, l \nu) n\left(k^{\prime} \mu^{\prime}, l^{\prime} \nu^{\prime}\right)\left(\delta_{k k^{\prime}} \delta_{\mu \mu^{\prime}} \delta_{l l^{\prime}} \delta_{\nu \nu^{\prime}}-\delta_{l k^{\prime}} \delta_{\nu \mu^{\prime}} \delta_{k l^{\prime}} \delta_{\mu \nu^{\prime}}(-1)^{j_{k}+j_{l}-J}\right),
\end{aligned}
$$

the unitary-matrix $\mathcal{U}$ in Eq. (5) is just unity-matrix and the matrix equation (5) simplifies to QRPA with proton-neutron pairing equation (full-QRPA) 17, 18, 33.

It is well-known that the QBA violates the Pauli exclusion principle because we have neglected terms coming from the commutator. This deficiency could be improved with help of renormalized quasi-boson approximation (RQBA) 


$$
\begin{aligned}
& {\left[A_{\mu \nu}(k, l, J, M), A_{\mu^{\prime} \nu^{\prime}}^{+}\left(k^{\prime}, l^{\prime}, J, M\right)\right]} \\
& \simeq\left\langle 0_{R P A}^{+}\left|\left[A_{\mu \nu}(k, l, J, M), A_{\mu^{\prime} \nu^{\prime}}^{+}\left(k^{\prime}, l^{\prime}, J, M\right)\right]\right| 0_{R P A}^{+}\right\rangle \\
& =n(k \mu, l \nu) n\left(k^{\prime} \mu^{\prime}, l^{\prime} \nu^{\prime}\right)\left(\delta_{k k^{\prime}} \delta_{\mu \mu^{\prime}} \delta_{l l^{\prime}} \delta_{\nu \nu^{\prime}}-\delta_{l k^{\prime}} \delta_{\nu \mu^{\prime}} \delta_{k l^{\prime}} \delta_{\mu \nu^{\prime}}(-1)^{j_{k}+j_{l}-J}\right) \\
& \times \underbrace{\left\{1-\frac{1}{\hat{\jmath}_{l}}<0_{R P A}^{+}\left|\left[a_{\nu l}^{+} a_{\nu i}\right]_{00}\right| 0_{R P A}^{+}>-\frac{1}{\hat{\jmath}_{k}}<0_{R P A}^{+}\left|\left[a_{\mu k}^{+} a_{\mu \tilde{k}}\right]_{00}\right| 0_{R P A}^{+}>\right\}}_{=: \mathcal{D}_{\mu k, \nu l ; J^{\pi}}},
\end{aligned}
$$

with the notations $\hat{\jmath}_{k}=\sqrt{2 j_{k}+1}$ and

$$
\left[a_{\mu k}^{+} a_{\mu \tilde{k}}\right]_{00}=\sum_{m_{k}} C_{j_{k} m_{k} j_{k}-m_{k}}^{00} a_{\mu k m_{k}}^{+} a_{\mu k-m_{k}}
$$

If $\mathcal{D}_{\mu k, \nu l ; J^{\pi}}=1$, one recovers the usual boson commutation relations for the QBA. The reduction of $\mathcal{D}_{\mu k, \nu l ; J^{\pi}}$ from 1 reflects the fact that one has the commutation relations of Fermion pairs (Pauli principle) and not of bosons.

It is useful to introduce renormalized operators

$$
\bar{A}_{\mu \nu}^{+}(k, l, J, M)=\mathcal{D}_{\mu k, \nu l ; J \pi}^{-1 / 2} A_{\mu \nu}^{+}(k, l, J, M)
$$

In the RQBA the overlap matrix $\mathcal{U}$ is also diagonal: $\mathcal{U}=D^{1 / 2}\left(\begin{array}{ll}1 & 0 \\ 0 & 1\end{array}\right) D^{1 / 2}$. After the transformations

$$
\begin{aligned}
\overline{\mathcal{A}} & =\mathcal{D}^{-1 / 2} \mathcal{A D}^{-1 / 2}, \quad \overline{\mathcal{B}}=\mathcal{D}^{-1 / 2} \mathcal{B D}^{-1 / 2}, \\
\bar{X}^{m} & =\mathcal{D}^{1 / 2} X^{m}, \quad \bar{Y}^{m}=\mathcal{D}^{1 / 2} Y^{m},
\end{aligned}
$$

for the phonon operator we can write

$$
Q_{J M^{\pi}}^{m+}=\sum_{\mu k \leq \nu l}\left\{\bar{X}_{\mu \nu}^{m}\left(k, l, J^{\pi}\right) \bar{A}_{\mu \nu}^{+}(k, l, J, M)+\bar{Y}_{\mu \nu}^{m}\left(k, l, J^{\pi}\right) \tilde{\bar{A}}_{\mu \nu}(k, l, J, M)\right\}
$$

and the renormalized quasiparticle random phase approximation (full-RQRPA) equation takes the form of RPA-like equation

$$
\left(\begin{array}{ll}
\overline{\mathcal{A}} & \overline{\mathcal{B}} \\
\overline{\mathcal{B}} & \overline{\mathcal{A}}
\end{array}\right)_{J^{\pi}}\left(\begin{array}{l}
\bar{X}^{m} \\
\bar{Y}^{m}
\end{array}\right)_{J^{\pi}}=\Omega_{J^{\pi}}^{m}\left(\begin{array}{cc}
1 & 0 \\
0 & -1
\end{array}\right)\left(\begin{array}{l}
\bar{X}^{m} \\
\bar{Y}^{m}
\end{array}\right)_{J^{\pi}} .
$$

The matrix elements $\overline{\mathcal{A}}$ and $\overline{\mathcal{B}}$ are explicitly given by 


$$
\begin{aligned}
& \overline{\mathcal{A}}_{J^{\pi}}\left(\mu k, \nu l ; \mu^{\prime} k^{\prime}, \nu^{\prime} l^{\prime}\right) \\
& =n(k \mu, l \nu) n(\dot{k} \dot{\mu}, \hat{l} \dot{\nu})\left\{\left(E_{k \mu}+E_{l \nu}\right)\left(\delta_{k \dot{k}} \delta_{\mu \dot{\mu}} \delta_{l i} \delta_{\nu \dot{\nu}}-(-1)^{j_{k}+j_{l}-J} \delta_{k i} \delta_{\mu \dot{\nu}} \delta_{l \dot{k}} \delta_{\nu \dot{\mu}}\right)\right. \\
& -2 \mathcal{D}_{\mu k, \nu l ; J^{\pi}}^{1 / 2} \mathcal{D}_{\mu^{\prime} k^{\prime}, \nu^{\prime} l^{\prime} ; J^{\pi}}^{1 / 2} \\
& \times \sum_{\alpha \beta \delta \gamma}\left[G(k \alpha l \beta \hat{k} \gamma \hat{l} \delta J)\left(u_{k \mu \alpha} u_{l \nu \beta} u_{\hat{k} \dot{\mu} \gamma} u_{\dot{l} \hat{\nu} \delta}+v_{k \mu \alpha} v_{l \nu \beta} v_{\hat{k} \dot{\mu} \gamma} v_{i \dot{l} \delta}\right)\right.
\end{aligned}
$$

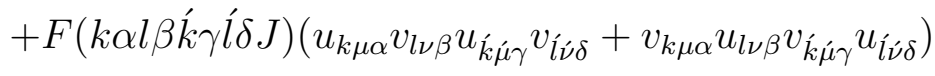

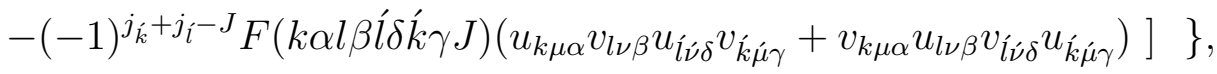

$$
\begin{aligned}
& \overline{\mathcal{B}}_{J^{\pi}}\left(\mu k, \nu l ; \mu^{\prime} k^{\prime}, \nu^{\prime} l^{\prime}\right) \quad=2 n(k \mu, l \nu) n\left(k \dot{k}, l^{\prime}\right) \mathcal{D}_{\mu k, \nu l ; J^{\pi}}^{1 / 2} \mathcal{D}_{\mu^{\prime} k^{\prime}, \nu^{\prime} l^{\prime} ; J^{\pi}}^{1 / 2} \\
& \times\left\{\sum _ { \alpha \beta \delta \gamma } \left[G(k \alpha l \beta \hat{k} \gamma \dot{l} \delta J)\left(u_{k \mu \alpha} u_{l \nu \beta} v_{\dot{k} \hat{\mu} \gamma} v_{l \dot{\nu} \delta}+v_{k \mu \alpha} v_{l \nu \beta} u_{\dot{k} \dot{\mu} \gamma} u_{i \dot{l} \delta \delta}\right)\right.\right. \\
& -F(k \alpha l \beta \hat{k} \gamma \dot{l} \delta J)\left(u_{k \mu \alpha} v_{l \nu \beta} v_{\hat{k} \dot{\mu} \gamma} u_{\dot{l} \dot{\nu} \delta}+v_{k \mu \alpha} u_{l \nu \beta} u_{\hat{k} \dot{\mu} \gamma} v_{l \dot{l} \delta}\right)
\end{aligned}
$$

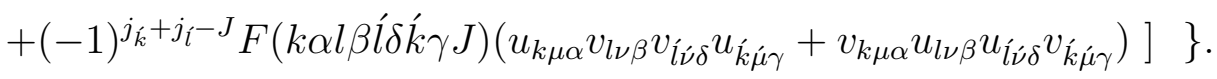

Here, $G(a \alpha b \beta c \gamma d \delta J)$ and $F(a \alpha b \beta c \gamma d \delta J)$ are respectively particle-particle and particle-hole interaction matrix-elements defined in [35]. The greek letters $\alpha, \beta, \gamma$ and $\delta$ are isospin indices (e.g., $\alpha=p, n$ ) while the greek letters $\mu, \mu^{\prime}, \nu$ and $\nu^{\prime}$ run the value 1 or 2 and denote the "quasi-isospin" of the quasiparticles.

In order to calculate the RQRPA matrices $\overline{\mathcal{A}}_{J^{\pi}}$ and $\overline{\mathcal{B}}_{J^{\pi}}$ one has to determine quantity $\mathcal{D}_{\mu k, \nu l ; J \pi}$. We follow the method of ref. [31] and express the one-body densities of Eq. (10) in terms of mappings

$$
\left[a_{\mu k}^{+} a_{\mu \tilde{k}}\right]_{00}=\frac{1}{\hat{j}_{k}} \sum_{\substack{\nu l \\ J M}} A_{\mu \nu}^{+}(k, l, J, M) A_{\mu \nu}(k, l, J, M) .
$$

By using eqs. (10), (12), (13), (18) together with

$$
\bar{A}_{\mu \nu}^{+}(k, l, J, M)=\sum_{m}\left\{\bar{X}_{\mu \nu}^{m}\left(k, l, J^{\pi}\right) Q_{J M^{\pi}}^{m+}-\bar{Y}_{\mu \nu}^{m}\left(k, l, J^{\pi}\right) \tilde{Q}_{J M^{\pi}}^{m}\right\},
$$

we obtain the following expression for $\mathcal{D}_{\mu k, \nu l ; j \pi}$ : 


$$
\begin{aligned}
\mathcal{D}_{k \mu l \nu J^{\pi}}=1- & \frac{1}{\hat{\jmath}_{k}^{2}} \sum_{\substack{k^{\prime} \mu^{\prime} \\
J^{\prime \pi^{\prime}} m}} \mathcal{D}_{k \mu k^{\prime} \mu^{\prime} J^{\prime \pi^{\prime}}} \hat{J}^{\prime 2}\left|\bar{Y}_{\mu \mu^{\prime}}^{m}\left(k, k^{\prime}, J^{\prime \pi^{\prime}}\right)\right|^{2} \\
& -\frac{1}{\hat{\jmath}_{l}^{2}} \sum_{\substack{l^{\prime} \nu^{\prime} \\
J^{\prime \prime} m}} \mathcal{D}_{l \nu l^{\prime} \nu^{\prime} J^{\prime \pi^{\prime}}} \hat{J}^{\prime 2}\left|\bar{Y}_{\nu \nu^{\prime}}^{m}\left(l, l^{\prime}, J^{\prime \pi^{\prime}}\right)\right|^{2}
\end{aligned}
$$

The system of non-linear equations in Eq. (20) could be solved numerically by an iteration process. As input the solution of the full-RQRPA equations for all the considered multipolarities is required. However, in order to calculate the full-RQRPA equation in Eq. (15) we need the knowledge of the renormalization factors $\mathcal{D}_{k \mu l \nu J \pi}$. Therefore, the selfconsistent scheme of the calculation is a doubly iterative problem. As starting values for $\mathcal{D}_{k \mu l \nu J \pi}$ for the first iteration could be used e.g.,

$$
\mathcal{D}_{k \mu l \nu J^{\pi}}=1-\frac{1}{\hat{\jmath}_{k}^{2}} \sum_{\substack{k^{\prime} \mu^{\prime} \\ J^{\prime} \pi_{m}^{\prime} m}} \hat{J}^{\prime 2}\left|Y_{\mu \mu^{\prime}}^{m}\left(k, k^{\prime}, J^{\prime \pi^{\prime}}\right)\right|^{2}-\frac{1}{\hat{\jmath}_{l}^{2}} \sum_{\substack{l^{\prime} \nu^{\prime} \\ J^{\prime \prime} \pi_{m}}} \hat{J}^{\prime 2}\left|Y_{\nu \nu^{\prime}}^{m}\left(l, l^{\prime}, J^{\prime \pi^{\prime}}\right)\right|^{2},
$$

with the amplitudes $Y^{m}$ being the solution of the full-QRPA equation.

The half-life for $2 \nu \beta \beta$-decay could be written in a factorized form [1]- [5]:

$$
\left(T_{1 / 2}^{2 \nu \beta \beta}\right)^{-1}=G_{G T}^{2 \nu}\left|M_{G T}^{2 \nu}\right|^{2},
$$

where $G_{G T}^{2 \nu}$ is a lepton phase space integral and the nuclear matrix elements $M_{G T}$ in the full-RQRPA is

$$
M_{G T}^{2 \nu}=\sum_{m_{i}, m_{f}} \frac{{ }_{f}<0_{R P A}^{+}\left\|\tau^{+} \sigma\right\| 1_{m_{f}}^{+}><1_{m_{f}}^{+} \mid 1_{m_{i}}^{+}><1_{m_{i}}^{+}\left\|\tau^{+} \sigma\right\| 0_{R P A}^{+}>_{i}}{\Omega_{1^{+}}^{m_{f}}+\Omega_{1^{+}}^{m_{i}}}
$$

with reduced transition-amplitudes of the Gamow-Teller beta decay operator

$$
\begin{aligned}
& <1_{m_{i}}^{+}\left\|\tau^{+} \sigma\right\| 0_{R P A}^{+}>_{i}=\sum_{\mu k \leq \nu l} \sigma(k, l)\left(\mathcal{D}_{k \mu l \nu 1^{+}}^{i}\right)^{1 / 2} \\
& \times\left[\bar{X}_{\mu \nu}^{m_{i}}\left(k, l, 1^{+}\right)\left(u_{k \mu p}^{i} v_{l \nu n}^{i}-v_{k \mu n}^{i} u_{l \nu p}^{i}\right)+\bar{Y}_{\mu \nu}^{m_{i}}\left(k, l, 1^{+}\right)\left(v_{k \mu p}^{i} u_{l \nu n}^{i}-u_{k \mu n}^{i} v_{l \nu p}^{i}\right)\right], \\
& f<0_{R P A}^{+}\left\|\tau^{+} \sigma\right\| 1_{m_{f}}^{+}>=\sum_{\mu k \leq \nu l} \sigma(k, l)\left(\mathcal{D}_{k \mu l \nu 1^{+}}^{f}\right)^{1 / 2} \\
& \times\left[\bar{X}_{\mu \nu}^{m_{f}}\left(k, l, 1^{+}\right)\left(v_{k \mu p}^{f} u_{l \nu n}^{f}-u_{k \mu n}^{f} v_{l \nu p}^{f}\right)+\bar{Y}_{\mu \nu}^{m_{f}}\left(k, l, 1^{+}\right)\left(u_{k \mu p}^{f} v_{l \nu n}^{f}-v_{k \mu n}^{f} u_{l \nu p}^{f}\right)\right] .
\end{aligned}
$$

Here, $\sigma(k, l)=<k\|\sigma\| l>$ is the reduced matrix element of $\sigma$ operator. The HFB $u$ and $v$ factors entering in eqs. (24) and (25) belong to two different even-even system. The 
occupation amplitudes $u^{i}, v^{i}$ and $u^{f}, v^{f}$ are obtained by solving the HFB equation for the initial and final nuclear states, respectively. $\left|1_{m_{i}}^{+}\right\rangle$and $\left|1_{m_{f}}^{+}\right\rangle$are respectively the excited states calculated from the ground state of initial $(\mathrm{A}, \mathrm{Z})$ and final $(\mathrm{A}, \mathrm{Z}+2)$ nuclei with the excitation energies $\Omega_{1^{+}}^{m_{f}}$ and $\Omega_{1^{+}}^{m_{i}}$. The states $\mid 1_{m_{i}}^{+}>\left(\mid 1_{m_{f}}^{+}>\right)$are expressed in terms of

forwards- $\bar{X}_{\mu \nu}^{m_{i}}\left(k, l, 1^{+}\right)\left(\bar{X}_{\mu \nu}^{m_{f}}\left(k, l, 1^{+}\right)\right)$and backwards- $\bar{Y}_{\mu \nu}^{m_{i}}\left(k, l, 1^{+}\right)\left(\bar{Y}_{\mu \nu}^{m_{f}}\left(k, l, 1^{+}\right)\right)$going amplitudes. Unfortunately the states $\mid m_{i}, 1^{+}>$and $\mid m_{f}, 1^{+}>$are not orthogonal to each other. For the overlap matrix of these states we write

$$
<1_{m_{f}}^{+} \mid 1_{m_{i}}^{+}>=\sum_{\mu k \leq \nu l}\left(\bar{X}_{\mu \nu}^{m_{i}}\left(k, l, 1^{+}\right) \bar{X}_{\mu \nu}^{m_{f}}\left(k, l, 1^{+}\right)-\bar{Y}_{\mu \nu}^{m_{i}}\left(k, l, 1^{+}\right) \bar{Y}_{\mu \nu}^{m_{f}}\left(k, l, 1^{+}\right)\right) .
$$

We note that in the limit of small ground state correlations $\mathcal{D}_{k \mu l \nu J^{\pi}} \simeq 1$ the full-RQRPA could be replaced by the full-QRPA. The deviation between the results of both methods is a signal showing on the restriction of the validity of the QBA in a given physical region. Further, in the limit in which there is no proton-neutron paring, the full-QRPA reduces to two matrix equations [17, 18, 33]: The first pp+nn QRPA equation has the origin in the proton-proton and neutron-neutron quasiparticle excitations. The second pn-QRPA equation considers only the proton-neutron quasiparticle excitations from the ground state. By neglecting the proton-neutron pairing interaction the full-RQRPA equation decomposes in a similar way on pp+nn RQRPA and pn-RQRPA equations. However, pp+nn RQRPA and pn-RQRPA equations remain coupled through the Eq. (20). Therefore, both equations have to be solved parallel in the iterative process.

\section{CALCULATION AND DISCUSSION OF THE RESULTS}

We applied the full-RQRPA method to the $2 \nu \beta \beta$-decay of ${ }^{76} \mathrm{Ge},{ }^{82} \mathrm{Se},{ }^{128} \mathrm{Te}$ and ${ }^{130} \mathrm{Te}$. We assumed the single particle model space both for protons and neutrons as follows.

(i) For ${ }^{76} \mathrm{Ge} \rightarrow{ }^{76} \mathrm{Se}$ and ${ }^{82} \mathrm{Se} \rightarrow{ }^{82} \mathrm{Kr}$ the model space comprises 13 levels:

$$
1 s_{1 / 2}, 0 d_{5 / 2}, 0 d_{3 / 2}, 1 p_{3 / 2}, 1 p_{1 / 2}, 0 f_{7 / 2}, 0 f_{5 / 2}, 2 s_{1 / 2}, 1 d_{5 / 2}, 1 d_{3 / 2}, 0 g_{9 / 2}, 0 g_{7 / 2}, 0 h_{11 / 2}
$$

(i) For ${ }^{128} \mathrm{Te} \rightarrow{ }^{128} \mathrm{Xe}$ and ${ }^{130} \mathrm{Te} \rightarrow \rightarrow^{130} \mathrm{Xe}$ we used 16 levels: 


$$
\begin{gathered}
1 p_{3 / 2}, 1 p_{1 / 2}, 0 f_{7 / 2}, 0 f_{5 / 2}, 2 s_{1 / 2}, 1 d_{5 / 2}, 1 d_{3 / 2}, 0 g_{9 / 2}, 0 g_{7 / 2}, 0 h_{11 / 2}, 0 h_{9 / 2}, 1 f_{7 / 2}, 1 f_{5 / 2}, \\
2 p_{3 / 2}, 2 p_{1 / 2}, 0 i_{13 / 2}
\end{gathered}
$$

The single particle energies have been calculated with a Coulomb-corrected Wood-Saxon potential. For the two body interaction we used the nuclear G-matrix calculated from Bonn one-boson exchange potential. The single quasiparticle energies and occupation amplitudes have been found by solving the HFB equation with p-n pairing for both the parent and the daughter nuclei in the above mentioned space. The renormalization of the protonproton, neutron-neutron and proton-neutron pairing interaction has been determined according to ref. [17, 18]. The renormalization of the $\mathrm{T}=1 \mathrm{~J}=0$ proton-neutron force is due to the quadrupole deformation of the intrinsic nucleus. It could also be described in a spherical basis by admixing to the single particle propagator $2^{+}$quadrupole excitations. Thus this renormalization and the HFB step influence only the quasiparticle states. The collective excitations are described in a second QRPA step. The renormalization we fit to the proton-neutron pairing gap [17, 18].

In the calculation of the full-RQRPA equation we renormalized particle- particle and particle- hole channels of the G-matrix interaction by introducing two parameters $g_{p p}$ and $g_{p h}$, which in principle should be equal to unity. Since the matrix element $M_{G T}^{2 \nu}$ is much more sensitive to $g_{p p}$ than to $g_{p h}$, we will set $g_{p h}=1.0$ and discuss the dependence on $g_{p p}$. Only for the $2^{+}$-channel of the interaction $g_{p h}$ was fixed to $g_{p h}=0.8$ for all studied $2 \nu \beta \beta$-decay transitions. For higher value of $g_{p h}$ the particle-hole interaction in $2^{+}$channel is too strong. The lowest eigenvalue becomes the imaginary and leads to a collapse of the correlated ground state.

It is worthwhile mentioning that the calculation of $M_{G T}^{2 \nu}$ within full-RQRPA needs a great computational effort. We remind that the full-RQRPA self- consistent scheme of the calculation requires the solution of the full-RQRPA equation (15) for all multipolarities $J^{\pi}$ in each iteration for the initial and the final nuclei. In comparison with the full-RQRPA, the full-QRPA calculation of $M_{G T}$ requires to solve the full-QRPA equation only for the 
multipolarity $1^{+}$once for initial and once for final nucleus. The iterative procedure of the full-RQRPA have been found to converge rapidly.

As the full-RQRPA calculation is a very time consuming process, it is interesting to determine, which multipolarities $J^{\pi}$ are important to take into account to calculate the renormalization factors $\mathcal{D}_{\mu k, \nu l ; J^{\pi}}$ in Eq. (20). We made a calculation for $g_{p p}=1.0$ including more and more multipolarities $J^{\pi}$ in the renormalization scheme of the full-RQRPA. The results are shown in Fig. 1. Reading from the left to the right, the quoted multipolarity is added at every point to the ones of the preceding point. One can clearly see a saturation effect for large multipolarities. This can be explained by the fact that the higher multipolarities are less collective and do only little contribute to the ground state correlations. Practically, one is able to find a combination of the multipolarities, that optimizes both the effect of renormalization and the effort of the computing-time. Fortunately, it turns out that one combination, which consists of the $1^{+}, 2^{+}, 2^{-}$and $3^{-}$multipolarities, shows as good results as the whole set. The corresponding values of $M_{G T}^{2 \nu}$ for the studied nuclear transitions are plotted with empty symbols in Fig. 1. A given combination of the multipolarities have been used to study the $g_{p p}$ dependence of the matrix element $M_{G T}^{2 \nu}$.

The goal of the present paper is to study the reliability of the QBA in the nuclear structure calculation of $M_{G T}^{2 \nu}$. This information can be obtained by the comparison of the results of the full-RQRPA and the full-QRPA in the physically acceptable region of the $g_{p p}$ parameters $0.8 \leq g_{p p} \leq 1.2$. Our results for $2 \nu \beta \beta$-decay of ${ }^{76} G e,{ }^{82} \mathrm{Se},{ }^{128} \mathrm{Te}$ and ${ }^{130}$ Te are presented in Fig. 2, Fig. 3, Fig. 4 and Fig. 5, respectively. We see that the effect due to the ground state correlations beyond the RPA is improving the agreement for all studied nuclear transitions. The inclusion of the ground state correlations beyond RPA was found to stabilize the behaviour of $M_{G T}^{2 \nu}$ as a function of $g_{p p}$. The full-QRPA and the full-RQRPA equations are of the same form, differing only by a renormalization of the two-body interaction by the factor $\mathcal{D}_{\mu k, \nu l ; J^{\pi}}$ [see Eq. (15)]. For $\mathcal{D}_{\mu k, \nu l ; J^{\pi}} \simeq 1$, what is the case for small ground state correlations, the results of both methods are the same. However, the QRPA quenching mechanism for $M_{G T}^{2 \nu}$ has its origin in generating too much 
ground state correlations with increasing strength of the particle-particle interaction. Strong ground state correlations lead to a significantly smaller value of $\mathcal{D}_{\mu k, \nu l ; J^{\pi}}$ and reduces the strength of two-body interaction. In this way a damping of the dependence of $M_{G T}^{2 \nu}$ on $g_{p p}$ is obtained. The full-RQRPA has been found to reproduce well the value of $M_{G T}^{2 \nu-e x p}$ deduced from the experimental half-live. Agreement is achieved for ${ }^{76} \mathrm{Ge}$ and ${ }^{82} \mathrm{Se}$ for the parameters $g_{p p} \simeq 0.95$ and $g_{p p} \simeq 1.00$, respectively. In the case of $2 \nu \beta \beta$-decay of ${ }^{128} T e$ and ${ }^{130} T e$ we need higher values of the renormalization parameter $g_{p p}: g_{p p} \simeq 1$. We remind that the Te isotopes are known to be a very special and delicate case. W. Haxton already found this out [36. It could be connected with the fact that the two Te isotopes 128 and 130, for which the double beta decay is measured, are unharmonic or even slightly deformed, so that a spherical RPA treatment as used here is not sufficient.

Both, the full-QRPA and full-RQRPA, include proton-neutron pairing correlations via the HFB method. In order to clarify the role of proton-neutron pairing in the stabilization of the dependence of $M_{G T}^{2 \nu}$ on $g_{p p}$, we switched off proton-neutron pairing interaction and performed the calculation within pn-QRPA and pn-RQRPA (no p-n pairing). The results are presented in Figs. 2-5. We can see that also in this case one can avoid a strong instability of $M_{G T}^{2 \nu}$. It is interesting to note that the effect due to the proton-neutron pairing is significant. The inclusion of proton-neutron pairing in the self-consistent scheme of the calculation is also helping to shift the instability of $M_{G T}^{2 \nu}$ on $g_{p p}$ to larger values of $g_{p p}$, which are outside the physical range $\left(0.8 \leq g_{p p} \leq 1.3\right)$.

\section{CONCLUSIONS AND OUTLOOK}

In summary, we have proposed a new higher (renormalized) QRPA many-body method for nuclear structure studies which we call full-RQRPA. We point out that in the full-RQRPA proton-neutron pairing is taken into account. The full-RQRPA includes the Pauli exclusion principle in a more proper way in comparison with the QRPA by using the renormalized quasi-boson approximation. The one-particle densities of the correlated state have been 
evaluated by following the approach of Catara et al. In this way the ground state correlations beyond the RPA are included in the self-consistent scheme of the calculation consisting of a set of non-linear equations in amplitudes $\mathrm{X}$ and $\mathrm{Y}$, which are solved by iteration.

We have employed the full-RQRPA to calculate $2 \nu \beta \beta$-decay of ${ }^{76} \mathrm{Ge},{ }^{82} \mathrm{Se},{ }^{128} \mathrm{Te}$ and ${ }^{130} \mathrm{Te}$. The nuclear matrix element $M_{G T}^{2 \nu}$ governing this process has been calculated. We have found $M_{G T}^{2 \nu}$ to be rather stable in the physically acceptable region of the parameter $g_{p p}$, which determine the strength of the particle-particle interaction. It allows us to predict more reliable values of the nuclear matrix elements. The relative large differences between the results of the full-RQRPA and the full-QRPA are related to violations of the Pauli exclusion principle by the second method due to ground state correlations. The quasiboson approximation is a poor approximation to study the $2 \nu \beta \beta$-decay. Therefore, an alternative methods should be elaborated in which the Pauli exclusion principle is explicitly incorporated. The shell model approach, which does not violate the Pauli exclusion principle, fails to construct all needed states of the intermediate nucleus. We note that the recently proposed two-vacua random phase approximation method could be perhaps a step in the right direction [37,38.

The renormalized QRPA which includes the Pauli effect of fermion pairs goes beyond the quasi-boson approximation. The inclusion of the Pauli principle reduces the ground state correlations and moves the collapse outside the physical region. Our matrix elements are derived from the Bonn-potential and if we would stick to them, $g_{p p}$ would be unity. Each RPA solution is collapsing eventually if the forces are made stronger and stronger. The point is that this collapse happens here only outside the physical region as in all stable RPA solutions and therefore the collapse disappeared for the description of the nuclei.

The full-RQRPA could find a wider use in the studies of other nuclear processes, e.g., single beta decay, neutrinoless double beta decay and pion double charge exchange reactions. 


\section{REFERENCES}

[1] W.C. Haxton and G.S. Stephenson Prog. Part. Nucl. Phys. 12 (1984) 409.

[2] M.G. Schepkin, Sov. Phys. Usp. 27 (1984) 555.

[3] M. Doi, T. Kotani and E. Takasugi, Prog. Theor. Phys. (Supp.) 83 (1985) 1.

[4] J.D. Vergados, Phys. Rep. 133 (1986) 1.

[5] T. Tomoda, Rep. Prog. Phys. 54 (1991) 53.

[6] P. Vogel, M.R. Zirnbauer, Phys. Rev. Lett. 57 (1986) 3148.

[7] O. Civitarese, A. Faessler, T. Tomoda, Phys. Lett. B 194 (1987) 11.

[8] K. Muto, H.V. Klapdor, Phys. Lett. B 201 (1988) 420.

[9] K. Muto E. Bender, and H.V. Klapdor, Z. Phys. A 334 (1988) 177.

[10] J.Engel, P. Vogel and M.R. Zirnbauer, Phys. Rev. C 37 (1988) 731.

[11] T. Tomoda and A. Faessler,Phys. Lett. B 199 (1987) 475.

[12] K. Muto, E. Bender and H.V. Klapdor, Z. Phys. A 334 (1989) 187.

[13] J. Engel, P. Vogel, O. Civitarese and M.R. Zirnbauer, Phys. Lett. B 208 (1988) 187.

[14] J. Suhonen, O. Civitarese and A. Faessler, Nucl. Phys. A 543 (1992) 645.

[15] G. Pantis, A. Faessler, W. Kaminski and J.D. Vergados, J. Phys. G 18, (1992) 605.

[16] F. Krmpotić, Phys. Rev. C 48 (1993) 1452.

[17] M.K. Cheoun, A. Bobyk, Amand Faessler F. Šimkovic and G. Teneva, Nucl. Phys. A $561(1993) 74$.

[18] M.K. Cheoun, A. Bobyk, A. Faessler, F. Šimkovic and G. Teneva, Nucl. Phys. A 564 (1993) 329. 
[19] A.A. Raduta, A. Faessler, S. Stoica, and W.A. Kaminski, Phys. Lett. B 254 (1991) 7.

[20] O. Civitarese, A. Faessler, J. Suhonen, and X.R. Wu, Nucl. Phys. A 524 (1991) 404.

[21] F. Krmpotić, A. Mariano, T.T.S. Kuo, and K. Nakayama, Phys. Lett. B 319 (1993) 393.

[22] K. Hara, Prog. Theor. Phys. 32 (1964) 88.

[23] K. Ikeda et al., Prog. Theor. Phys. 33 (1965) 22.

[24] D.J. Rowe, Rev. Mod. Phys. 40 (1968) 153,

D.J. Rowe, Nucl. Phys. A 107 (1968) 99.

[25] J. da Providencia, Nucl. Phys. A 108 (1968) 589.

[26] D.J. Rowe, Phys. Rev. 175 (1968) 1283.

[27] C.H. Johnson and C. Mahaux, Phys. Rev. C 38 (1988) 2589.

[28] H. Lenske and J. Wambach, Phys. Lett. B 249 (1990) 377.

[29] A. Klein et al., Nucl. Phys. A 535 (1991) 1.

[30] D. Karadjov, V.V. Voronov and F. Catara, Phys. Lett. B 306 (1993) 197.

[31] F. Catara, N. Dinh Dang and M. Sambataro, Nucl. Phys. A 579 (1994) 1.

[32] J. Toivanen and J. Suhonen, Phys. Rev. Lett. 75 (1995) 410.

[33] M.K. Cheoun, A. Faessler, F. Šimkovic, G. Teneva and A. Bobyk, Nucl. Phys. A 587 (1995) 301.

[34] A. Goswami, Nucl. Phys. 60 (1964) 228.

[35] M. Baranger, Phys. Rev. 120 (1960) 957.

[36] W.C. Haxton, Nucl. Phys. B (Proc. Supl.) 31 (1993) 88.

[37] F. Šimkovic, G. Teneva, A. Bobyk, S.B. Khadkikar and A. Faessler, Prog. Part. Nucl. 
Phys. 32 (1994) 329.

[38] G. Teneva, F. Šimkovic, A. Bobyk, M.K. Cheoun, A. Faessler and S.B. Khadkikar, Nucl. Phys. A 32 (1995) 249. 


\section{FIGURES}

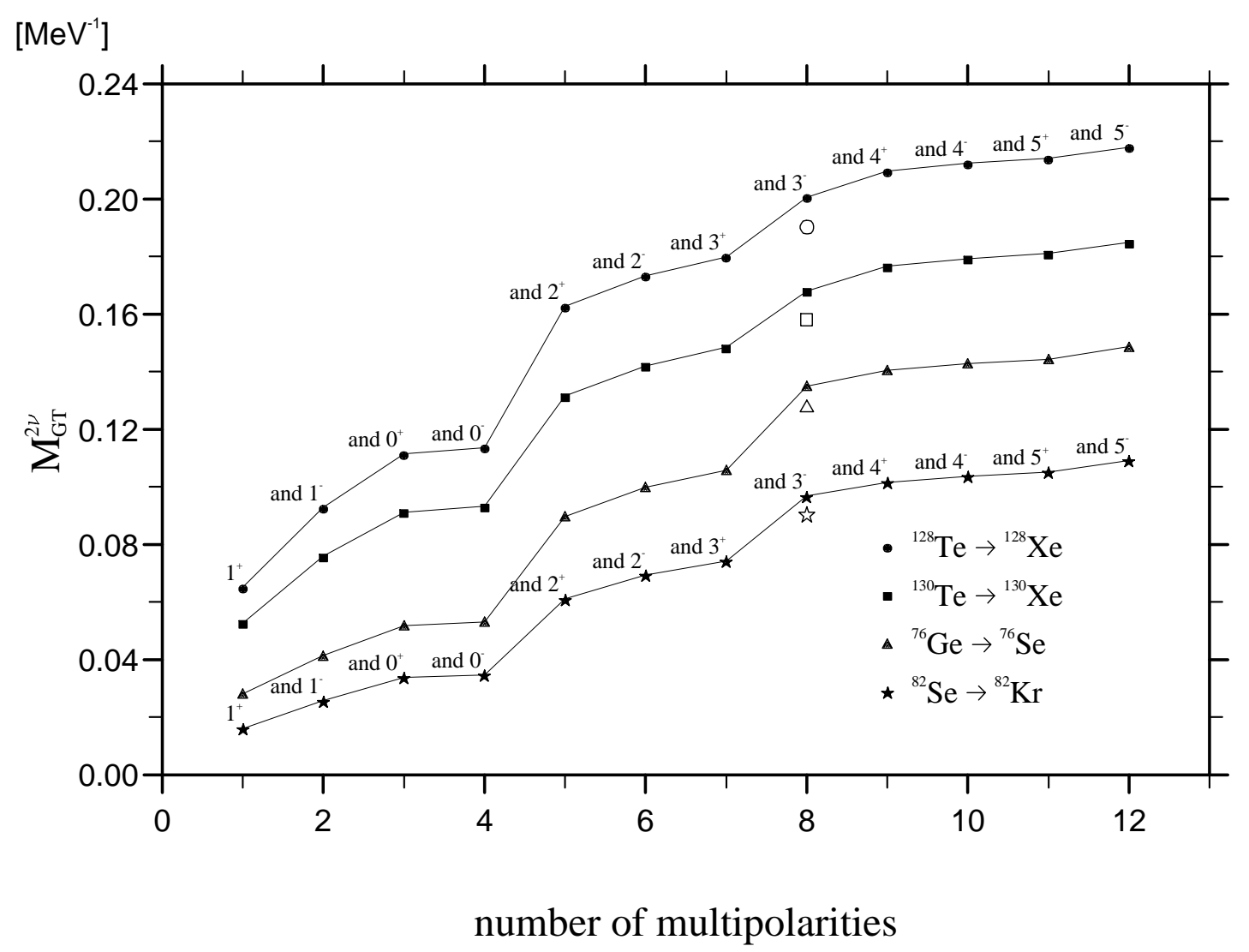

FIG. 1. The influence of including more and more multipolarities $J^{\pi}$ in the full-RQRPA calculation of $M_{G T}^{2 \nu}$ for $g_{p p}=1.0$. Starting from $1^{+}$the mentioned multipole is added to the former ones at every point. By this procedure the last point shows the value of $M_{G T}^{2 \nu}$ obtained by the consideration of 12 multipolarities ranging from $0^{+}$to $5^{-}$. The empty symbols mark the values for the combination of $1^{+}, 2^{+}, 2^{-}$and $3^{-}$multipolarities, which turn out to exhaust approximately the most of the correlations beyond RPA. 


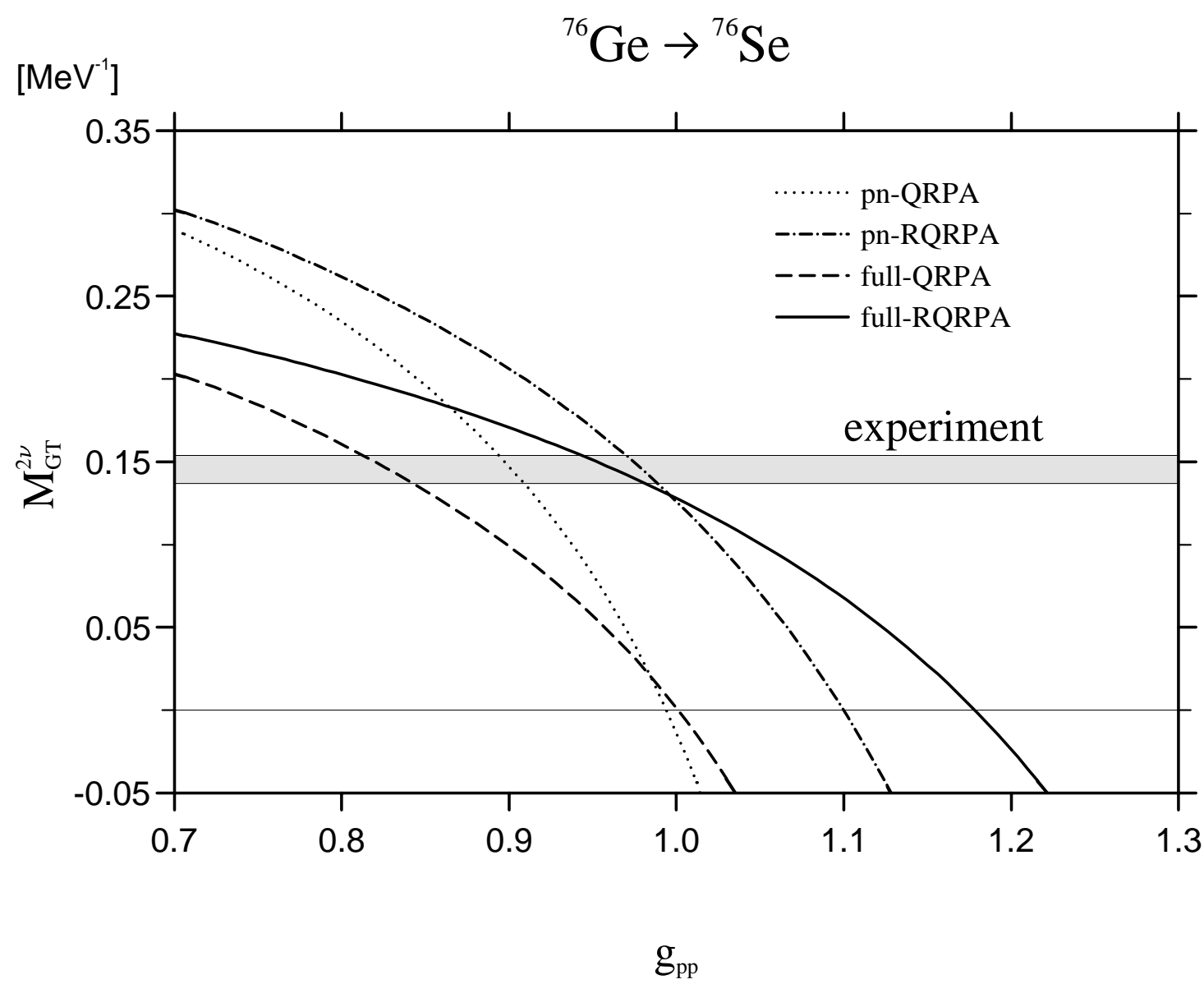

FIG. 2. The Gamow-Teller transition matrix element $M_{G T}^{2 \nu}$ of the $2 \nu \beta \beta$-decay of ${ }^{76} G e$ is plotted as function of particle-particle coupling constant $g_{p p}$. The solid line corresponds to full-RQRPA (with p-n pairing), the dashed line to full-QRPA (with p-n pairing), the dash-dotted line to pn-RQRPA (without p-n pairing) and the dotted line to pn-QRPA calculation (without p-n pairing), respectively. 


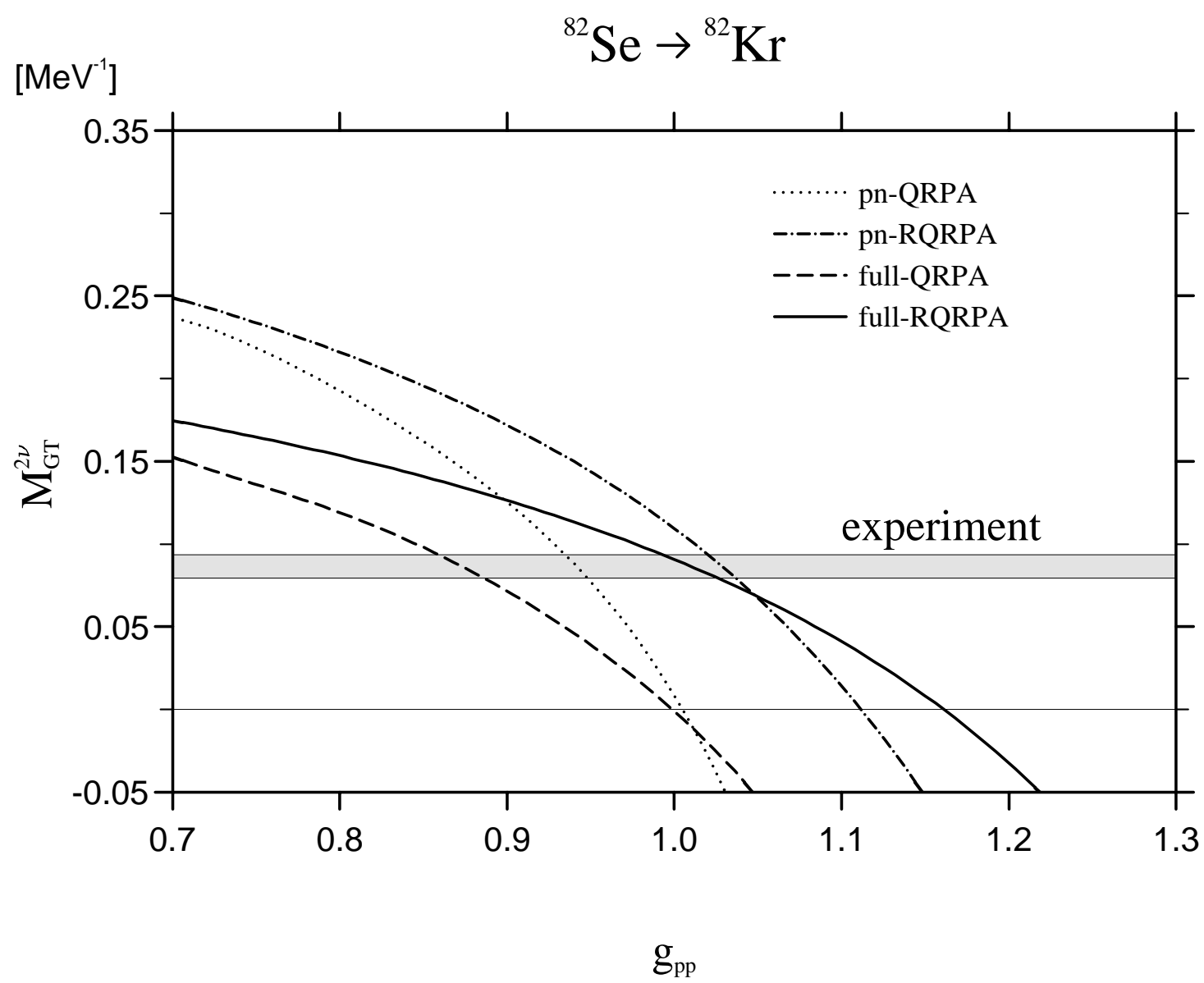

FIG. 3. The same as Fig. 2 for ${ }^{82} S e$. 


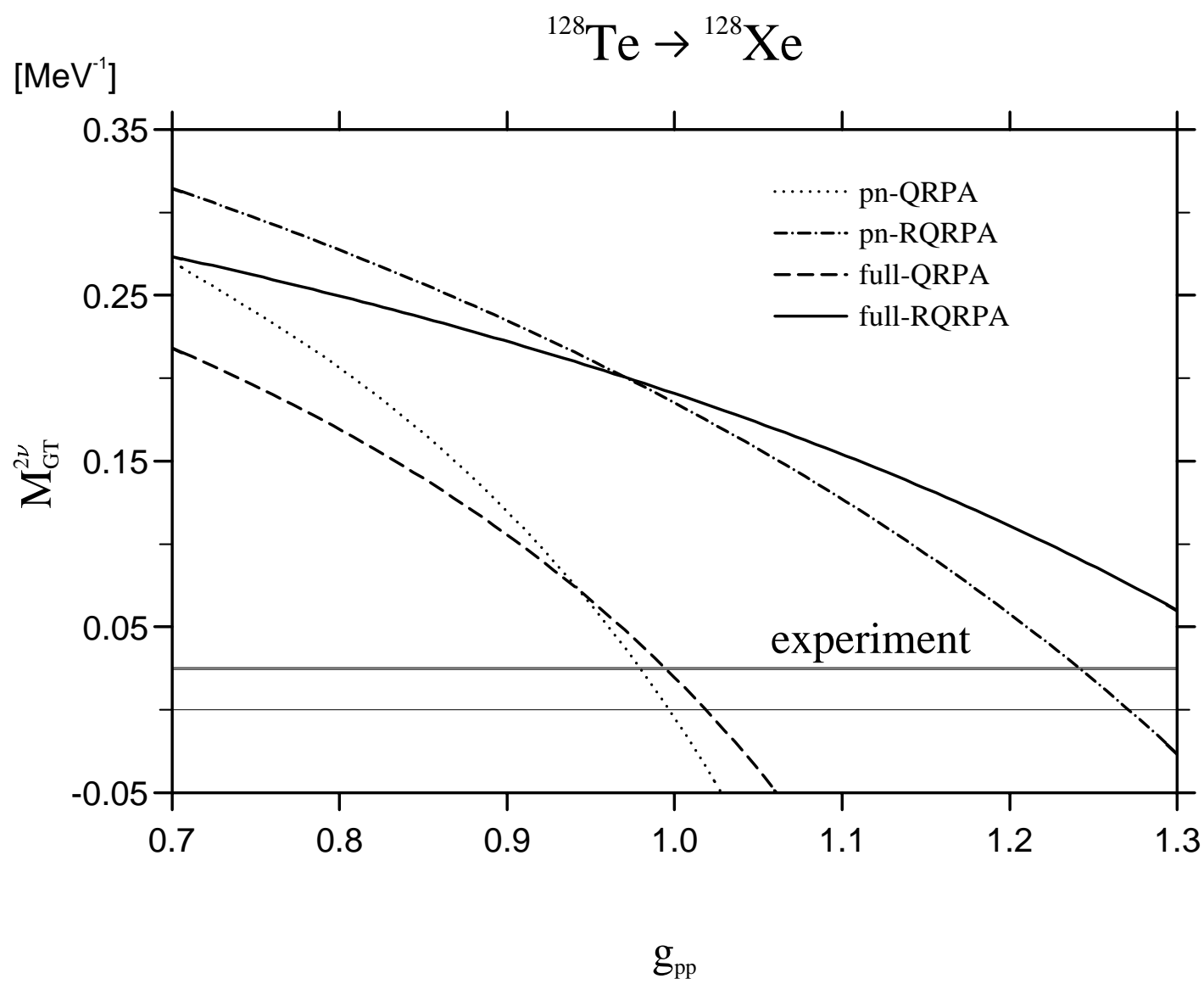

FIG. 4. The same as Fig. 2 for ${ }^{128}$ Te. 


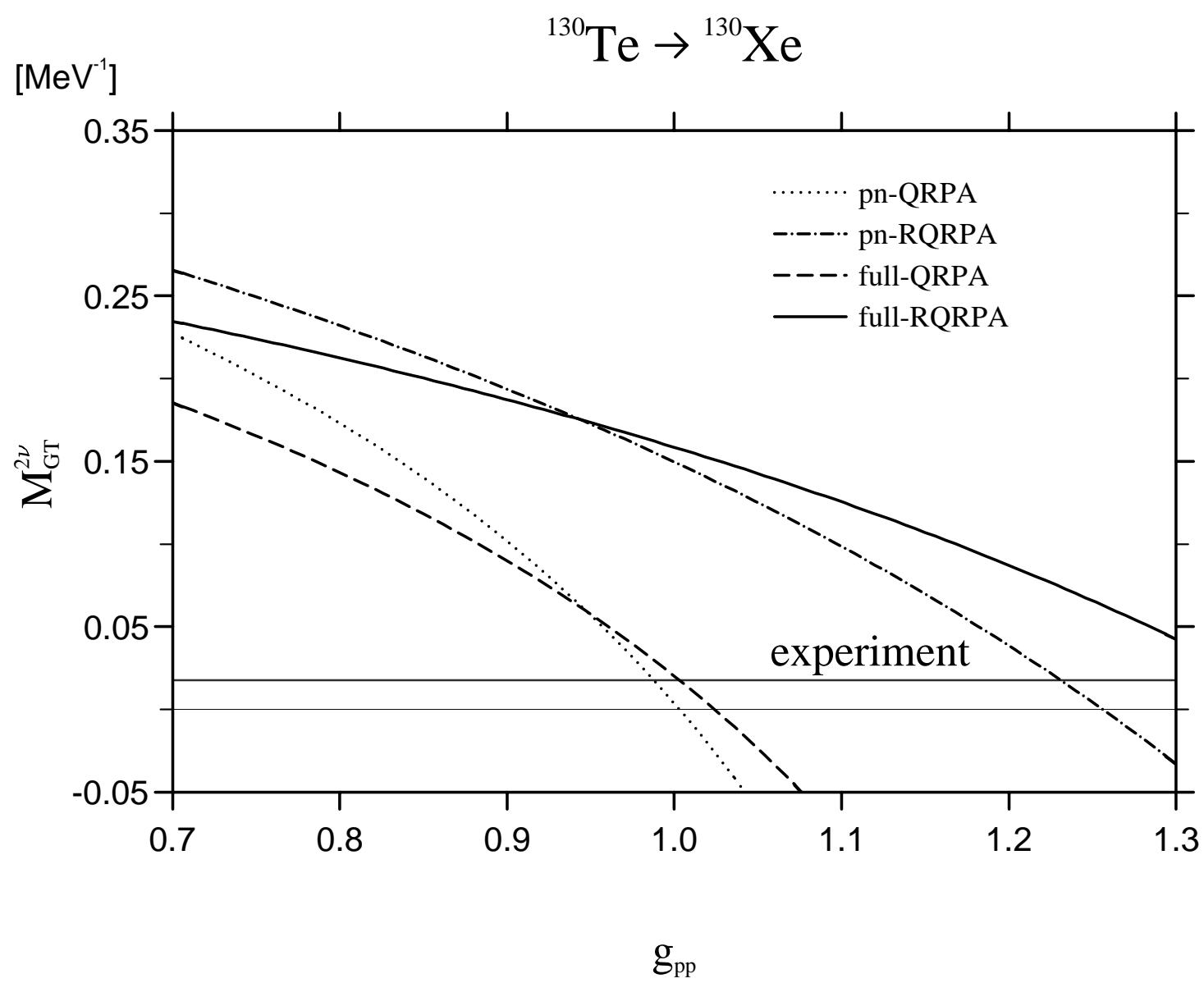

FIG. 5. The same as Fig. 2 for ${ }^{130} T e$. 